\title{
Achilles Tendoscopy for Non-Insertional Tendinopathy
}

\author{
Giovanni Carcuro, Leonardo Lagos, Hugo Henríquez, Natalio Cuchacovich, \\ Manuel Pellegrini, Christian Bastías
}

Foot and Ankle Unit, Instituto Traumatológico Teodoro Gebauer, Santiago, Chile.

Email: leo_lagos@yahoo.com

Received March 21 $1^{\text {st }}, 2013$; revised May $2^{\text {nd }}, 2013$; accepted May $28^{\text {th }}, 2013$

Copyright (C) 2013 Giovanni Carcuro et al. This is an open access article distributed under the Creative Commons Attribution License, which permits unrestricted use, distribution, and reproduction in any medium, provided the original work is properly cited.

\begin{abstract}
Achilles tendinopathy is a very common pathology, especially in the athletic population. Its etiology is multifactorial and the underlying pathophysiology is still not fully understood. For non-insertional tendinopathy is increasingly recognized the influence of paratendinopathy in the genesis of patients symptoms, so various surgical treatments have been accordingly developed; they are indicated when conservative treatment fails. The Achilles tendoscopy is one of these techniques, it has the advantages of minimally invasive surgery, resulting in a low complication rate and good functional results. In relation to this procedure we review the surgical technique, which is exemplified by the case of a high performance athlete treated satisfactorily.
\end{abstract}

Keywords: Achilles Tendinitis; Non-Insertional; Tendoscopy; Surgical Treatment; Tendinopathy

\section{Introduction}

The Achilles tendon is the most important motor unit of the lower leg and strongest tendon in the human body [1], it is composed of the Gastrocnemius and Soleus muscles, and it is lined by a paratenon which is divided into two layers: parietal and visceral. The tendon rotates $90^{\circ}$ so that the Soleus portion inserts anteromedial in the calcaneal tuberosity while the Gastrocnemius portion inserts posterolateral. A region located 2 to $6 \mathrm{~cm}$ proximal to its insertion is relatively avascular, making it prone to injury [2].

Achilles tendon pathology is very common, affecting mostly athletic population, but it also can be present in sedentary patients. The etiology of the dysfunction is multifactorial and has been found to be associated with overuse injuries, training error, malalignment of the lower extremity, inflammatory disorders, and intrinsic disease or degeneration [3].

Different classifications of the pathology exists, one of them divides it in insertional tendinopathy (around the tendon at its calcaneal insertion) and non-insertional tendinopathy (proximal to the calcaneal insertion) [2]. Other classification involves histological changes: peritendinitis, with pathological changes localized to the peritenon; peritendinitis with tendinosis, with involvement of peritendinous structures and tendon degeneration; and tendinosis, with asymptomatic degeneration of the tendon without concomitant inflammation [4].

The source of the pain in non-insertional tendinopathy has not been clarified. Many of the treatments, especially surgical treatment, address the degenerative changes of the tendon, but the tendon itself is aneuronal, so this histological findings does not explain completely the clinical manifestations. Neovascularization and neoinnervation from surrounding tissues are actually the more accepted theories about the origin of the pain in this patients $[5,6]$.

Non operative treatment is the first choice for Achilles non-insertional tendinopathy. Over $70 \%$ of good outcomes had been reported [7], and specifically eccentric exercises have the most evidence of effectiveness on the relief of symptoms [8]. Surgical treatment is indicated when a well run conservative treatment, for at least 3 to 6 months, fails [2]. Surgical alternatives over time have changed alongside a better understanding of the origin of the disease: initially open surgery was the only alternative and focused on the degenerative changes of the tendon; this approach has gradually given way to miniinvasive procedures, which focus on management of tendon and paratenon pathology as well, among which we can mention: percutaneous longitudinal tenotomies, consisting in multiple stab incisions made with a number 11 scalpel blade through the skin to the tendon in the area of maximum tenderness and swelling; minimally invasive 
tendon stripping, in which 4 skin incisions $(0.5 \mathrm{~cm}$ each) are made on each side of the tendon, two proximal and two distally located, and a suture is passed thru the anterior and posterior aspects of the tendon as a "Gigli saw" causing the tendon to be freed from adhesions; and endoscopic debridement as the tendoscopy technique we describe in this article [2,5]. The common goals of them are the denervation of the diseased tendon, lysis of tendon adhesion, and stimulation of healing response [1, 2,5].

As well as other endoscopic techniques for foot and ankle surgery, Achilles tendoscopy offers advantages compared with open surgery such as smaller wounds, fewer wound infections, less blood loss, less morbidity and quicker return to activity [5,9]. An arthroscopic shaving is used to release the tendon, debride adhesions within the paratenon and even with the plantaris tendon as described by some authors [10]. Good results with this technique has been reported in the literature $[9,10]$.

\section{Surgical Technique}

Achilles tendoscopy is performed using the technique described by Steenstra and van Dijk [9]. The patient is in a prone position with a tourniquet placed around the upper thigh. The affected foot is placed at the end of the table allowing the surgeon to move the foot in full range of motion. The zone of the lesion (nodule) is marked and two portals are placed: one located 2 to $4 \mathrm{~cm}$ proximal and medial to the tendon lesion; and the other 2 to $3 \mathrm{~cm}$ distal and lateral to the lesion. The distal portal is made first with an incision through the skin only. The crural fascia is penetrated using a mosquito clamp which is followed by the blunt obturator and then a $2.9-\mathrm{mm}$ endoscope is introduced. To facilitate proximal portal placement a spinal needle can be used. Once in this space between the tendon and paratenon, a shaver is placed and used for debridement of the fibrous tissue, and release of the adhesions within the paratenon and plantaris tendon. Using this technique, small nodules also can be debrided, and longitudinal tenotomies in the Achilles can be performed using the arthroscopy probe under direct visualization.

In the aftercare the patient can start range of motion and weight bearing exercises as tolerated immediately.

\section{Clinical Case}

Male, 29 years, professional football player.

During interseason training over synthetic pitch, he presents pain and crepitus on the posterior aspect of the ankle, specifically over Achilles mid-portion region. An ultrasound was performed, and the results show paratendinopathy without intratendinous disease (Figure 1). A treatment with NSAIDs and rehabilitation program was initiated, without the relief of the symptoms after 2 months. At this point, a peritendinous infiltration with 40 $\mathrm{ml}$ of saline solution with a low dose of corticosteroids under ultrasound was indicated (Figure 2); a daily rehabilitation program was maintained, evolving with complete relief of pain, but once training and impact activities were initiated, the pain and crepitus reappears.

Given the poor outcome of symptoms, a new ultrasound and MRI was performed, both of them show paratendinopathy signs without intratendinous changes (Figure 3).

It is decided to return to a rehabilitation program discarding impact activities; initially the patient evolved satisfactorily, but by increasing the intensity of physical labor symptoms recur. After 4 months of well done physical therapy the player shows no decrease on symp-

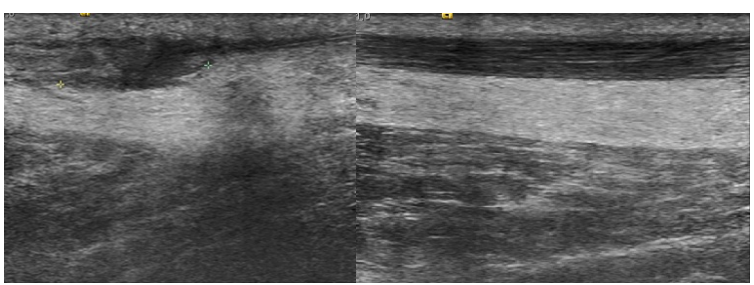

Figure 1. Ultrasound (US) shows paratendinopathy without tendon lesion.

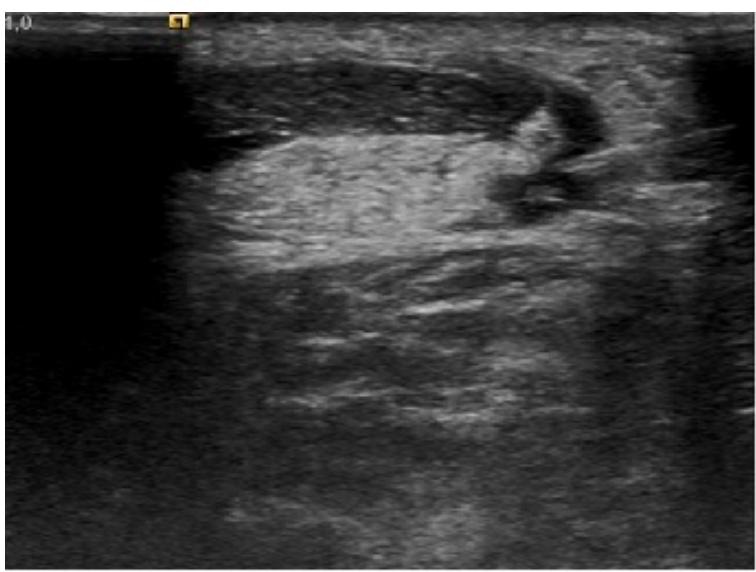

Figure 2. Infiltration of peritendinous space under US guidance.

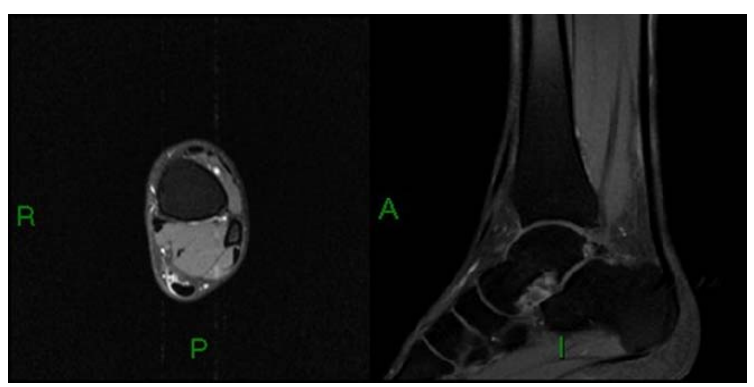

Figure 3. MRI shows paratendinopathy without tendinopathy. 
toms and present inability for competition, so surgical treatment was indicated. Achilles tendoscopy was chosen, because is a case of paratendinopathy without intratendinous lesions, and this endoscopic technique allows a good and direct visualization of all the peritenon adheons and the release of them.

During surgery some findings can be seen: synovitis and peritendinous adhesions (Figure 4(a)), and a great adhesion between Achilles and plantaris tendon (Figure 4(b)). Debridement of the adhesions between paratenon and Achilles tendon, and adhesions between the tendons was performed with a shaver (Figure 4(c)); finally a complete release of the tendon and debridement of hypertrophic synovium is achieved (Figure 4(d)).

In the aftercare the patient is discharged from hospital with a dressing and allowed for weight bearing and ankle mobilization as tolerated. After 3 days the dressing is changed and the patient starts rehabilitation program, which is mainly focused on strengthening and stretching of the Achilles tendon based on eccentric contraction exercises.

The patient evolved satisfactorily achieving impact activities without restriction at fourth week and returning completely to competition at sixth week.

\section{Discussion}

Achilles tendoscopy is an alternative for the management of patients with Achilles non-insertional tendinopathy when conservative treatment has failed, especially when there is a combination of tendinopathy and paratendinopathy [9]. Effectively offers the advantages of minimally

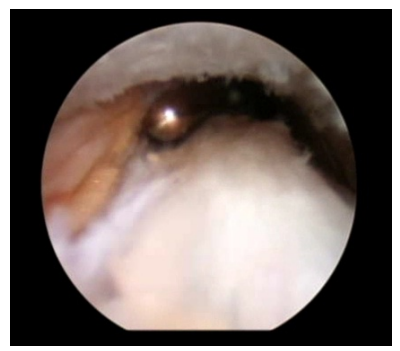

(a)

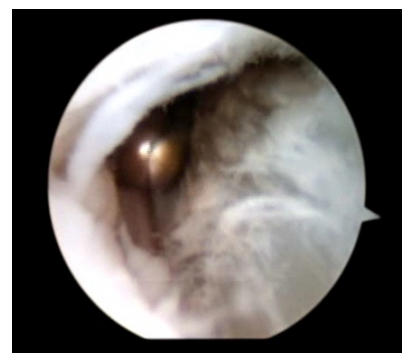

(c)

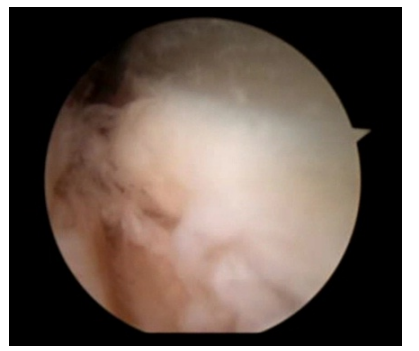

(b)

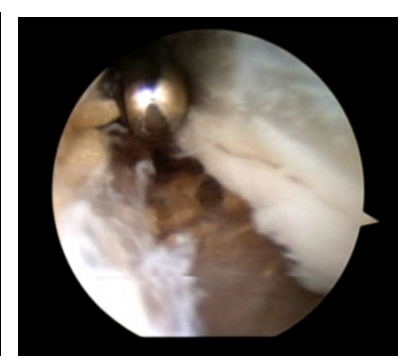

(d)
Figure 4. (a), (b) Tendoscopic view of adhesions and hypertrophic synovium; (c), (d) Debridement of the adhesions and release of the tendon. invasive surgery: lower complications and early return to activities.

Good outcomes have been reported in two studies in the literature. Steenstra and van Dijk treated 20 patients, and were followed up for a mean of 6 years; no complications were seen and all of them present a significant pain relief with AOFAS and SF-36 scores similar to a cohort of people without tendon complaints [9]. Pearce et al. reported similar results in 11 patients followed up for a mean of 2 years, no complications were seen, and 8 of the 11 patients were satisfied with the procedure, while the other 3 were somewhat satisfied [10]. These studies show satisfactory results, as seen in the clinical case we have shown, with no complications or late effects reported; but are no comparative studies with a limited number of patients, with a short to middle-term follow up, so there is a need for research with a higher level of evidence to objectify the role of this and other mini-invasive techniques in the treatment of Achilles non-insertional tendinopathy.

\section{REFERENCES}

[1] P. Phisitkul, "Endoscopic Surgery of the Achilles Tendon," Current Reviews in Musculoskeletal Medicine, Vol. 5, No. 2, 2012, pp. 156-163. doi:10.1007/s12178-012-9115-1

[2] G. Murphy, "Surgical Treatment of Non-Insertional Achilles Tendinitis," Foot and Ankle Clinics of North America, Vol. 14, No. 4, 2009, pp. 651-661. doi:10.1016/j.fcl.2009.08.008

[3] M. Krahe and G. Berlet, “Achilles Tendon Ruptures, Rerupture with Revision Surgery, Tendinosis, and Insertional Disease," Foot and Ankle Clinics of North America, Vol. 14, No. 4, 2009, pp. 247-275.

doi:10.1016/j.fcl.2009.04.003

[4] G. Puddu, E. Ippolito and F. Postachini, “A Classification of Achilles Tendon Disease," The American Journal of Sports Medicine, Vol. 4, No. 4, 1976, pp. 145-150. doi:10.1177/036354657600400404

[5] N. Maffulli, U. Longo, F. Oliva, M. Ronga and V. Denaro, "Minimally Invasive Surgery of the Achilles Tendon," Orthopedic Clinics of North America, Vol. 40, No. 4, 2009, pp. 491-498. doi:10.1016/j.ocl.2009.05.006

[6] M. Van Sterkenburg and C. Niek van Dijk, "Mid-Portion Achilles Tendinopathy: Why Painful? An Evidence-Based Philosophy," Knee Surgery, Sports Traumatology, Arthroscopy, Vol. 19, 2011, pp. 1367-1375. doi:10.1007/s00167-011-1535-8

[7] J. Lake and S. Ishikawa, "Conservative Treatment of Achilles Tendinopathy: Emerging Techniques,” Foot and Ankle Clinics of North America, Vol. 14, No. 4, 2009, pp. 663-674. doi:10.1016/j.fcl.2009.07.003

[8] R. Magnussen, W. Dunn and A. Thomson, "Nonoperative Treatment of Midportion Achilles Tendinopathy: A Systematic Review," Clinical Journal of Sport Medicine, Vol. 19 , No. 1, 2009, pp. 54-64. 
doi:10.1097/JSM.0b013e31818ef090

[9] F. Steenstra and C. Niek van Dijk, “Achilles Tendoscopy,” Foot and Ankle Clinics, Vol. 11, No. 2, 2006, pp. 429-438. doi:10.1016/j.fcl.2006.02.001

[10] C. Pearce, J. Carmichael and J. Calder, “Achilles Tend- inoscopy and Plantaris Tendon Release and Division in the Treatment of Non-Insertional Achilles Tendinopathy," Journal of Foot and Ankle Surgery, Vol. 18, No. 2, 2012, pp. 124-127. doi:10.1016/j.fas.2011.04.008 